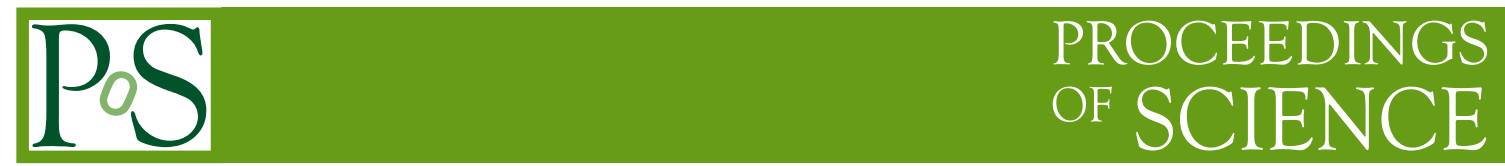

\title{
Circumbinary Disks
}

\section{Samuel Doolin}

Oxford University dept. Astrophysics

E-mail: sdoolin@astro.ox.ac.uk

\section{Katherine Blundell}

Oxford University dept. Astrophysics

E-mail: kmbeastro.ox.ac.uk

\begin{abstract}
We investigate the phenomenon of circumbinary disks: their stability and potentially insightful use as diagnostic tools of outflows. Our fully 3D simulations investigate the behaviour of circumbinary orbits, exploring binary mass fraction - eccentricity parameter space. The work presented also has implications for exoplanetary astronomy in the existence and determination of stable orbits in binary systems.
\end{abstract}

VII Microquasar Workshop: Microquasars and Beyond September 1 - 5, 2008

Foca, Izmir, Turkey 


\section{Astrophysical Context}

Figure 1 is a Very Long Baseline Array (VLBA) radio image of the famous microquasar SS433, displaying the characteristic "ruff" structure perpendicular to the radio jet axis. Blundell et al. [1] interpret this structure to be an equatorial outflow in the plane of the binary star system.

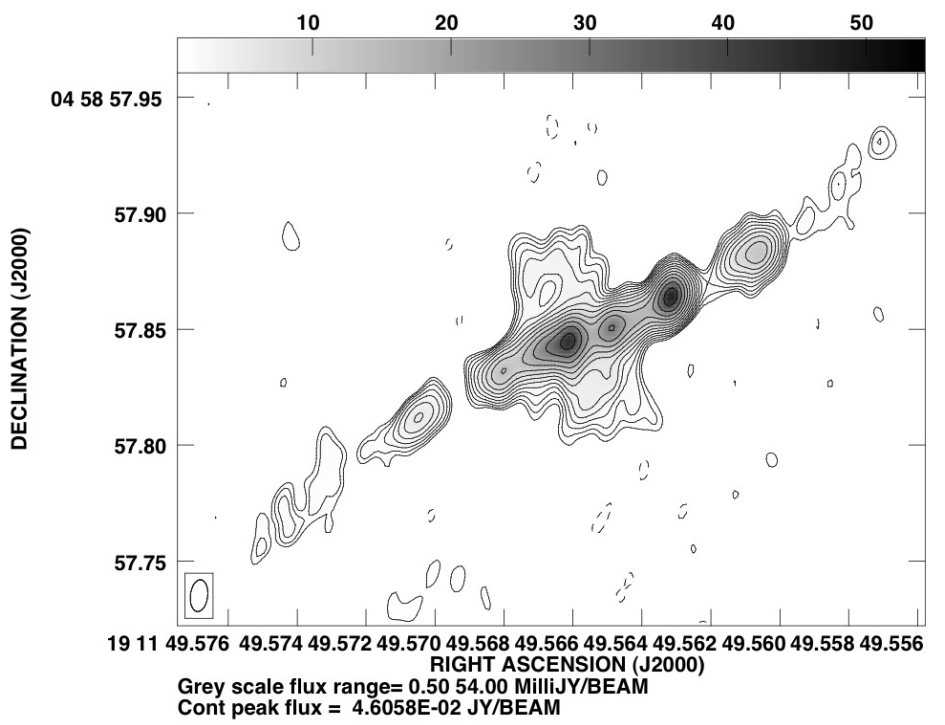

Figure 1: SS433. Blundell et al. 1, Fig. 2.

Through spectroscopic observations of SS433's Balmer $\mathrm{H} \alpha$ line, Blundell et al. [2] observe two narrow components of emission, constant in frequency. These components exhibit no orbital phase redshift variations and so are diagnosed as circumbinary in origin.

Blundell et al. [2] infer a circumbinary disk orbiting the binary system as a whole, and even suggest evidence for an accretion disk wind - circumbinary material interaction. Blundell et al. [2] calculate the system mass of SS433, obtaining the circumbinary disk orbital velocity from redshifts and the orbiting radius of the emitting material (the disk gap of the circumbinary disk) from numerical work by Holman and Wiegert [4]. This latter work investigated the stability of P-type (circumbinary) circular orbits. The "critical radius" is said to be the innermost radius at which a circular orbit remains stable, and this gives a good indication to the size of the disk gap.

\section{Method}

We follow and improve on Holman and Wiegert [4], numerically integrating a suite of noninteracting test particles around a binary of arbitrary eccentricity and mass ratio. We solve the binary directly by applying a dual bisection and Newton-Raphson algorithm to Kepler's equation.

We apply an adaptive step-size fourth and fifth-order Runge-Kutta integrator to integrate the system for $10^{4}$ binary orbital periods. During integration we remove test particles which come too close to either star, or escape the system. 


\section{Coplanar Results}

Our initial 2D coplanar results (Figure 2) broadly agree with Holman and Wiegert [4] and earlier work by Dvorak et al. [3], whilst improving resolution with more orbits investigated and a better sampling across binary mass ratio - eccentricity parameter space.

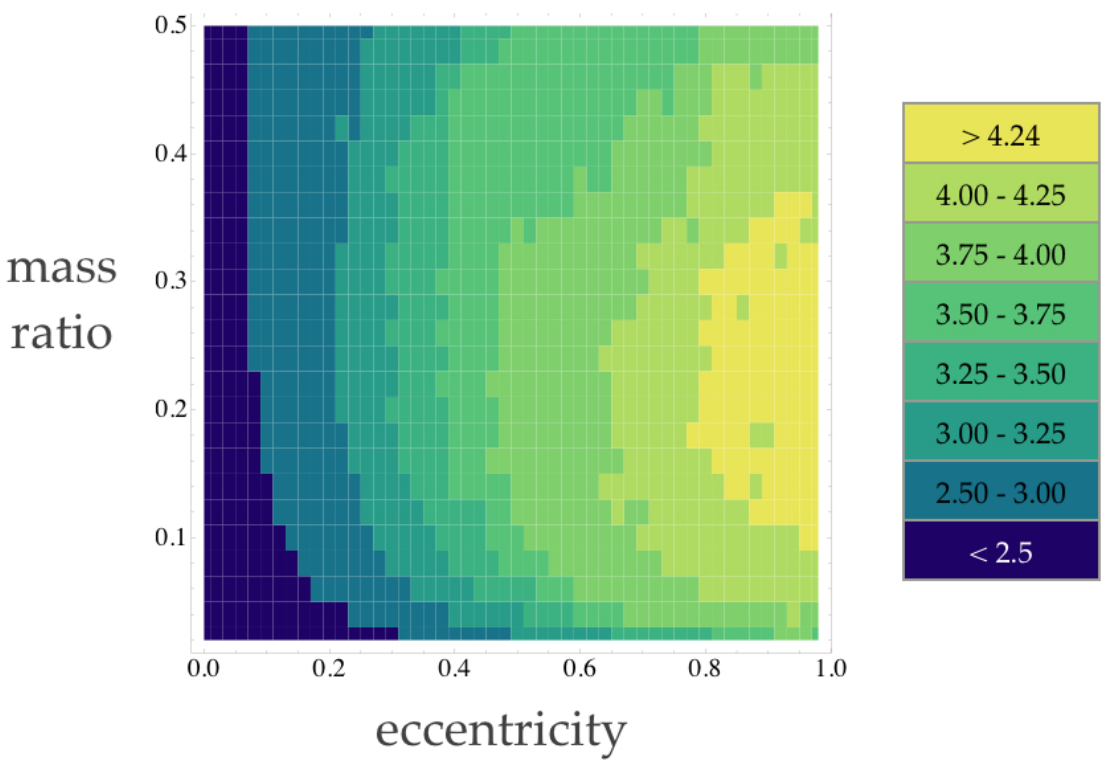

Figure 2: Coplanar results. Colour plot of critical radius (in units of binary semi-major axis) as a function of binary eccentricity and mass ratio.

\section{Improvements}

We next extend our simulations beyond Holman and Wiegert's into three dimensions, allowing us to investigate inclined circumbinary orbits as in Pilat-Lohinger et al. [5]. A general orbit in 3D about a point mass may be described by the Keplerian orbital elements $(a, e, i, W, w, v)$ as illustrated by Figure 3. Eccentricity $e$, semi-major axis $a$ and true anomaly $v$ describe the motion of a test particle in its orbital plane, whilst the inclination $i$, longitude of the ascending node $W$ and argument of perihelion $w$ describe the orientation of the orbital plane with respect to the plane of the binary. We track the orbital elements of each test particle about the centre of mass of the binary, outputting time-delayed snapshots to a MySQL database ${ }^{1}$. This method of handling orbital information has proven very effective - we retain unbiased data from our simulations, organise it effectively, and can ask very specific questions about orbital stability with SQL queries.

\footnotetext{
${ }^{1}$ These snapshots are of "osculating" orbits: the Keplerian orbit about a central body that a test particle would have if other perturbations were not present.
} 


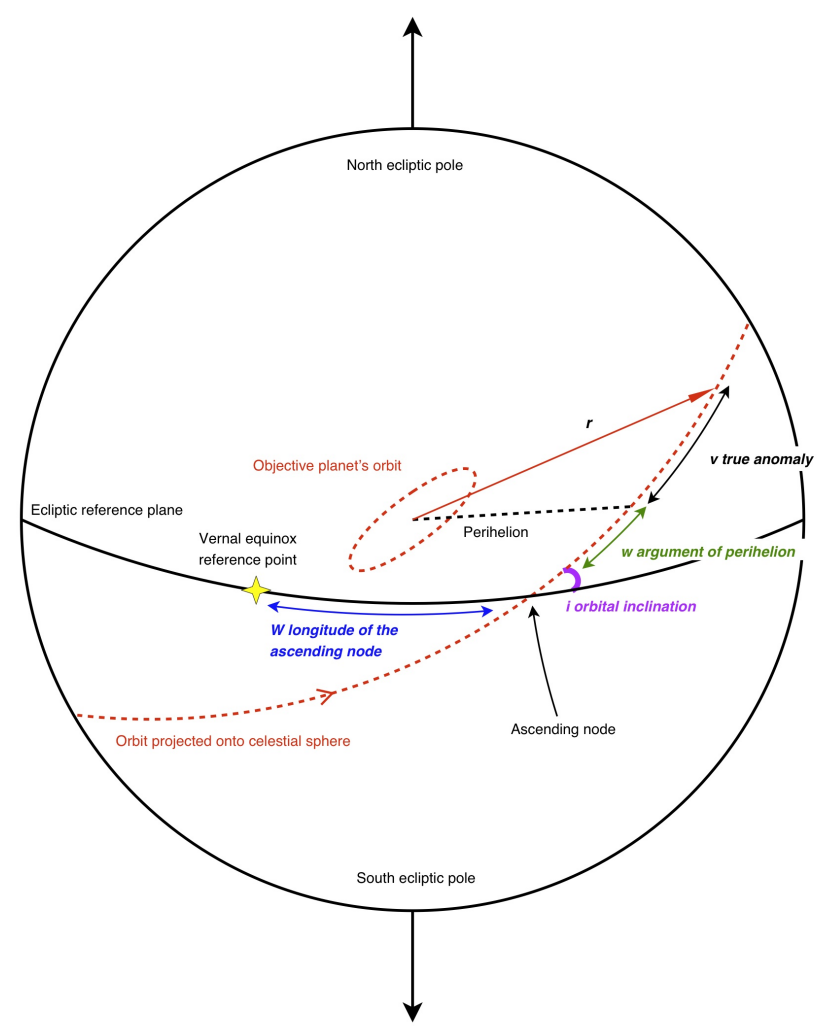

Figure 3: A representation of the Keplerian orbital elements
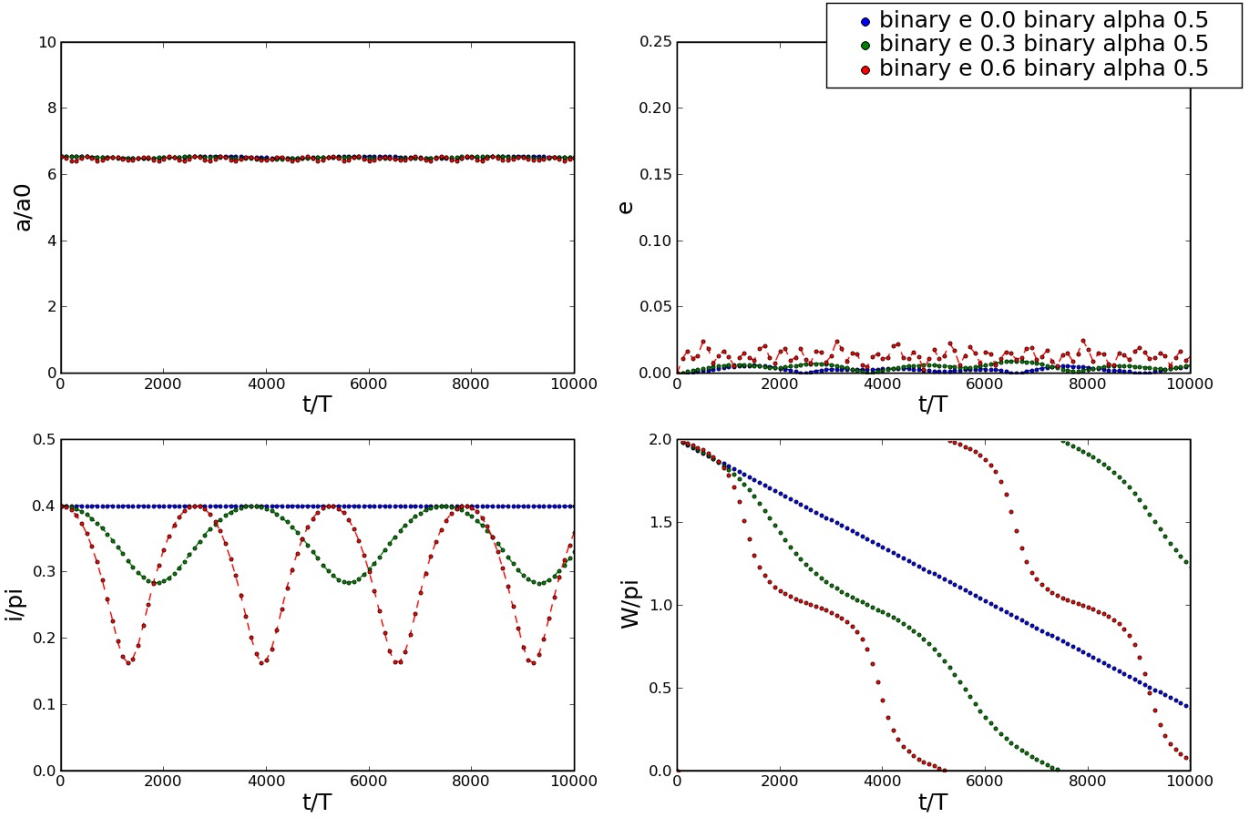

Figure 4: Tracking test particles: we examine specific test particles' orbital elements as a function of time. 


\section{Results}

Figure 4 is a plot of the orbital elements of an example test particle as a function of time. Three simulations are plotted; in each the test particle has the same initial conditions but experiences a different binary eccentricity. In all three cases the orbit has constant energy $(E \propto 1 / a)$ and remains essentially circular (zero eccentricity), but we see an orbital plane precession in the longitude of the ascending node $W$. This is a manifestation of the expected angular momentum exchange between the binary and the inclined test particle. We also note a wobble in the inclination $i$ of the orbital plane which increases in strength with the eccentricity of the binary. This could be viewed as a nutation in the processing angular momentum vector.

Figure 4 is but one example of many which exhibits some of the traits that we are beginning to recognise.

These orbits may be considered stable to an extent: although the orientation of the orbital plane changes over time with respect to the plane of the binary, the shape of the orbit remains constant, neither decaying nor expanding in the simulated $10^{4}$ binary periods. Further work will include the development of analytical tools to describe stablilty in the detailed behaviour of orbits around binary star systems revealed by our simulations.

\section{References}

[1] K. Blundell, A. Mioduszewski, T. Muxlow, P. Podsiadlowski and M. Rupen. Images of an equatorial outflow in SS433. The Astrophysical Journal, 562:L79, 2001.

[2] K. M. Blundell, M. G. Bowler and L. Schmidtobreick. SS 433: Observation of the circumbinary disk and extraction of the system mass. The Astrophysical Journal, 678:L47-L50, 2008.

[3] R. Dvorak, C. Froeschle and C. Froeschle. Stability of outer planetary orbits (p-types) in binaries. A\&A, 226(1):335-342, 1989.

[4] M. J. Holman and P. A. Wiegert. Long-term stability of planets in binary systems. The Astronomical Journal, 117:621 - 628, 1999.

[5] E. Pilat-Lohinger, B. Funk and R. Dvorak. Stability limits in double stars: a study of inclined planetary orbits. A\&A, 400:1085-1094, 2003. 